\title{
ON THE INDEPENDENCE THEOREM OF RELATED STRUCTURES FOR MODULAR (ARGUESIAN) LATTICES
}

\author{
G. GRÄTZER AND E. T. SCHMIDT
}

\begin{abstract}
Let $\mathfrak{D}$ be a finite distributive lattice with more than one element and let $\mathfrak{G}$ be a finite group. We prove that there exists a modular (arguesian) lattice $M$ such that the congruence lattice of $M$ is isomorphic to $\mathfrak{D}$ and the automorphism group of $M$ is isomorphic to $\mathfrak{G}$.
\end{abstract}

\section{INTRODUCTION}

The "related structures" of the title are the congruence lattice and the automorphism group of a lattice. In [4, Problem II. 18], the first author raised the problem whether the congruence lattice and the automorphism group of a finite lattice are independent. This problem was solved affirmatively by V. A. Baranskiǔ [1], [2] and A. Urquhart [11].

Both proofs are based on two characterization theorems:

(i) Every finite distributive lattice is isomorphic to the congruence lattice of a finite lattice (R. P. Dilworth, 1945, first published in [6]).

(ii) Every finite group is isomorphic to the automorphism group of a finite (distributive) lattice (G. Birkhoff [3]).

(There is a different approach to the Independence Theorem in [7] that does not rely on characterization theorems.)

In [10], the second author proved that every finite distributive lattice is isomorphic to the congruence lattice of a modular lattice (of course, no longer finite since the congruence lattice of a finite modular lattice is always Boolean). So it is natural to ask whether we could prove the Independence Theorem for modular lattices.

Independence Theorem for Modular Lattices. Let $\mathfrak{D}$ be a finite distributive lattice with more than one element and let $\mathfrak{G}$ be a finite group. Then there exists a modular lattice $M$ such that the congruence lattice of $M$ is isomorphic to $\mathfrak{D}$ and the automorphism group of $M$ is isomorphic to $\mathfrak{G}$.

The proof uses two constructions:

Theorem 1. Let $\mathfrak{G}$ be a finite group. Then there exists a simple modular lattice $S$ with an atom $p$ such that the automorphism group of $S$ is isomorphic to $\mathfrak{G}$ and every automorphism keeps $p$ fixed.

Date: Dec. 10, 1998.

1991 Mathematics Subject Classification. Primary 06B10; Secondary 08A05.

Key words and phrases. congruence lattice, automorphism group, modular, arguesian.

The research of the first author was supported by the NSERC of Canada.

The research of the third author was supported by the Hungarian National Foundation for Scientific Research, under Grant No. T29525. 
Theorem 2. Let $\mathfrak{D}$ be a finite distributive lattice with more than one element. There exists a rigid modular lattice $R$ with zero 0 and a dual atom $q$ such that the congruence lattice of $R$ is isomorphic to $\mathfrak{D}$.

Recall that a lattice $R$ is rigid, if it does not have a nontrivial automorphism.

Theorem 1 was proved in [8] without the statement about the atom; in Section 2, we modify the construction of [8] to obtain the form of the result we need in this paper. In Section 3, we prove Theorem 2 and, in Section 4, the Independence Theorem for Modular Lattices.

In Section 5, we prove a much stronger form of the Independence Theorem: We construct an arguesian lattice $A$ with a given finite congruence lattice and finite automorphism group. To accomplish that, we need Theorems 1 and 2 for arguesian lattices. In fact, in [8], we did that for Theorem 1. In Section 5, we prove this stronger form of Theorem 2 and show how the two arguesian theorems can be combined to get the Arguesian Independence Theorem.

\section{Simple MOdular LATTICES}

In [8], the construction starts by representing the finite group $\mathfrak{G}$ as the automorphism group of a finite graph $\langle V, E\rangle$. The reader should find it evident that if $\langle V, E\rangle$ has the property stated in the next lemma, then the modular lattice we construct does have the atom required in Theorem 1.

Lemma 1. Every finite group $\mathfrak{G}$ can be represented as the automorphism group of a finite graph $\langle V, E\rangle$ with the property:

There is a non-isolated vertex $f \in V$ fixed by all the automorphisms of $\langle V, E\rangle$.

Proof. Let $\left\langle V_{1}, E_{1}\right\rangle$ be a finite graph whose automorphism group is isomorphic to $\mathfrak{G}$. Form $\langle V, E\rangle$ as the disjoint union of $\left\langle V_{1}, E_{1}\right\rangle$ with a connected rigid finite graph $\langle R, S\rangle$ with a vertex $f \in R$ of degree $>\left|V_{1}\right|$. An automorphism $\alpha$ of $\langle V, E\rangle$ cannot take $f$ into $V_{1}$ since the degree of a vertex of $\left\langle V_{1}, E_{1}\right\rangle$ is $\leq\left|V_{1}\right|$. Hence $\alpha$ takes $f$ into $R$. Since $\langle R, S\rangle$ is connected, $\alpha$ takes $R$ into $R$. By the rigidity of $\langle R, S\rangle, \alpha$ is the identity map on $R$. So $\langle V, E\rangle$ has the same automorphism group as $\left\langle V_{1}, E_{1}\right\rangle$, and $f$ is fixed by all the automorphisms.

\section{Rigid MODUlar LATTICES}

In this section, we prove Theorem 2 ; the proof is based on the construction in [10]. For every finite poset $P$, we have to construct a rigid modular lattice $R_{P}$ with a dual atom $q$ such that Con $R_{P} \cong \mathbf{2}^{P}$.

We shall use the following lemma (the first six statements are due to the second author, see [10]; the last statement is due to A. Mitchke and R. Wille [9]).

Lemma 2. Let $E$ be a chain with bounds $0_{E}$ and $1_{E}$, and let $M_{3}=\{0, a, b, c, 1\}$ be the five-element nondistributive modular lattice. Let $M_{3}[E]$ denote the subposet of $E^{3}$ consisting of all $\langle x, y, z\rangle$ satisfying $x \wedge y=y \wedge z=z \wedge x$. Then the following statements hold:

(i) $M_{3}[E]$ is a modular lattice.

(ii) The subset

$$
\bar{M}_{3}=\{\langle 0,0,0\rangle,\langle 1,0,0\rangle,\langle 0,1,0\rangle,\langle 0,0,1\rangle,\langle 1,1,1\rangle\}
$$

of $M_{3}[E]$ is a sublattice of $M_{3}[E]$ and it is isomorphic to $M_{3}$. 
(iii) The subposet $\bar{E}=\{\langle x, 0,0\rangle \mid x \in E\}$ of $M_{3}[E]$ is a chain and it is isomorphic to $E$; we identify $E$ with $\bar{E}$.

(iv) $\bar{M}_{3}$ and $E$ generate $M_{3}[E]$.

(v) The subset

$$
\{\langle x, x \wedge y, y\rangle \mid x, y \in E\}
$$

of $M_{3}[E]$ is a sublattice and it is isomorphic to $E^{2}$.

(vi) Let $\Theta$ be a congruence relation of $E=\bar{E}$; then there is a unique congruence $\bar{\Theta}$ of $M_{3}[E]$ such that $\bar{\Theta}$ restricted to $\bar{E}$ is $\Theta$; therefore, Con $M_{3}[E] \cong$ Con $E$.

(vii) Let $M$ be a modular lattice; let $A=\{o, a, b, c, i\}$ be a sublattice of $M$ isomorphic to $M_{3}$; let $B$ be a chain in $[o, a]$ (including o and a) isomorphic to $E$. Then the sublattice of $M$ generated by $A$ and $B$ is isomorphic to $M_{3}[E]$.

The lattice $R_{P}$ will be put together by gluing together lattices of the form $M_{3}[E]$ with distributive lattices. The following lemma states some well-known facts about gluing and congruences.

Lemma 3. Let $A$ be a lattice with dual ideal $D$ with more than one element, and let $B$ be a lattice with ideal $I$ with more than one element. Let $D$ and $I$ be isomorphic under the isomorphism $\varphi$. Let $L$ denote the gluing of $A$ and $B$ over $D$ and $I$ with respect to $\varphi$.

(i) If $\Theta$ is a congruence of $L$, then $\Theta_{A}$ (the restriction of $\Theta$ to $A$ ) is a congruence of $A$, and $\Theta_{B}$ (the restriction of $\Theta$ to $B$ ) is a congruence of $B$, and $\Theta_{A}$ restricted to $D$ is the same as $\Theta_{B}$ restricted to $I$ under $\varphi$.

(ii) If $\Phi_{A}$ is a congruence of $A$ and $\Phi_{B}$ is a congruence of $B$ such that $\Phi_{A}$ restricted to $D$ is the same as $\Phi_{B}$ restricted to $I$ under $\varphi$, then there is a unique congruence $\Phi$ of $L$ such that the restriction of $\Phi$ to $A$ is $\Phi_{A}$, and the restriction of $\Phi$ to $B$ is $\Phi_{B}$.

(iii) If every congruence $\Phi$ of $B$ has the property that $\Phi_{I}$ (the restriction of $\Phi$ to $I$ ) has a unique extension to $B$ (namely, $\Phi$ ) and $\Phi_{I}$ (as a congruence of $D$ ) has an extension to a congruence of $A$, then $\operatorname{Con} L \cong \operatorname{Con} A$.

(iv) If $B$ and $I$ are simple, then $\operatorname{Con} L \cong \operatorname{Con} A$.

We now combine Lemmas 2 and 3 to construct the basic building block, see Figure 1.

Lemma 4. Let $U$ be a chain with zero, $0_{U}$, and unit, $1_{U}$. Let $0_{U} \leq u<v \leq 1_{U}$, and let $V$ be the interval $[u, v]$ of $U$. Construct the lattice $L$ as follows:

(i) Form the direct product $U \times V$.

(ii) Glue the interval $\left[\left\langle 0_{U}, u\right\rangle,\langle u, v\rangle\right]$ of $U \times V$ with the dual ideal $[\langle u, u\rangle,\langle u, v\rangle]$ $(\cong V)$ to $M_{3}[V]$ with the ideal $\{\langle 0,0, v\rangle \mid v \in V\}(\cong V)$.

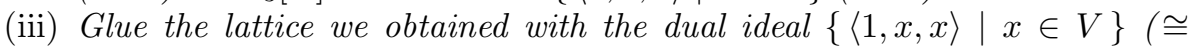
$V)$ to the interval $\left[\langle v, u\rangle,\left\langle 1_{U}, v\right\rangle\right]$ with the ideal $[\langle v, u\rangle,\langle v, v\rangle](\cong V)$; the resulting lattice is denoted by $U \circledast V$.

(iv) Consider $U$ as a sublattice of $U \circledast V$ by identifying $x \in U$ with $\langle x, u\rangle$ if $x \leq u$ or if $v \leq x$; otherwise, $x \in[u, v]$, and we identify $x$ with $\langle x, 0,0\rangle$. This identifies $U$ with a principal ideal of $U \circledast V$.

Then every congruence $\Theta$ of $U$ has a unique extension to a congruence $\bar{\Theta}$ of $U \circledast V$; therefore, Con $U \cong \operatorname{Con}(U \circledast V)$. 


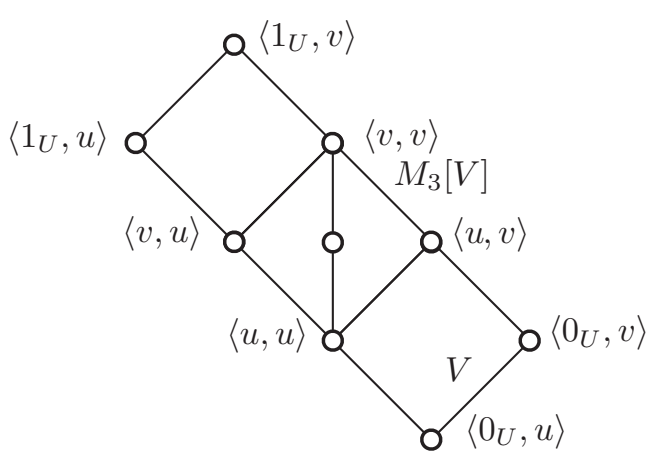

Figure 1. The lattice $U$.

We use induction on $n=|P|$, the size of $P$, to construct a rigid modular lattice $R_{P}$ with the following properties:

( $\left.I_{1}\right) R_{P}$ is a rigid modular lattice with zero $0_{P}$, unit $1_{P}$, and a dual atom $q_{P}$;

$\left(I_{2}\right)$ every principal congruence of $R_{P}$ is a join of join-irreducible congruences and the join-irreducible congruences of $R_{P}$ form a poset isomorphic to $P$;

$\left(I_{3}\right) R_{P}$ has an element $a_{P}$ with a unique complement $a_{P}^{\prime}$ such that the ideals $\left(a_{P}\right]$ and $\left(a_{P}^{\prime}\right]$ are isomorphic to a countable successor ordinal $\alpha_{P}$.

$\left(I_{4}\right)$ every congruence of $R_{P}$ is determined by its restriction to $\left(a_{P}^{\prime}\right]$.

Observe that $\left(I_{4}\right)$ is equivalent to the following condition:

$\left(I_{4}^{\prime}\right) R_{P}$ contains a chain

$$
0_{P} \leq b_{1}<c_{1} \leq b_{2}<c_{2} \leq \cdots \leq b_{n}<c_{n} \leq a_{P}^{\prime}
$$

such that the join-irreducible congruences of $R_{P}$ are exactly the principal congruences $\Theta\left(b_{1}, c_{1}\right), \Theta\left(b_{2}, c_{2}\right), \ldots, \Theta\left(b_{n}, c_{n}\right)$.

If $P=\mathbf{1}$ (that is, $|P|=1$ ), then the lattice of Figure 2 satisfies these requirements with $\alpha_{1}=4$; this lattice is also simple and rigid.

Now let $P$ be a finite poset, and let us assume that $R_{S}$ has been constructed for all finite posets $S$ satisfying $|S|<|P|$. The reader may find it useful to follow the induction step with Figure 3, which illustrates the construction of $R_{\mathbf{2}}(P=\mathbf{2}$, the two-element chain); the congruence lattice of $R_{2}$ is, of course, the three-element chain, $\mathbf{2}^{2}$.

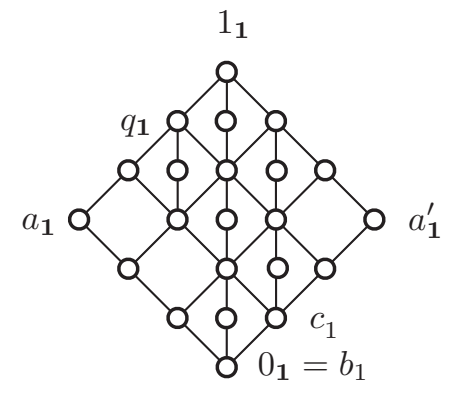

Figure 2. The lattice $R_{1}$. 


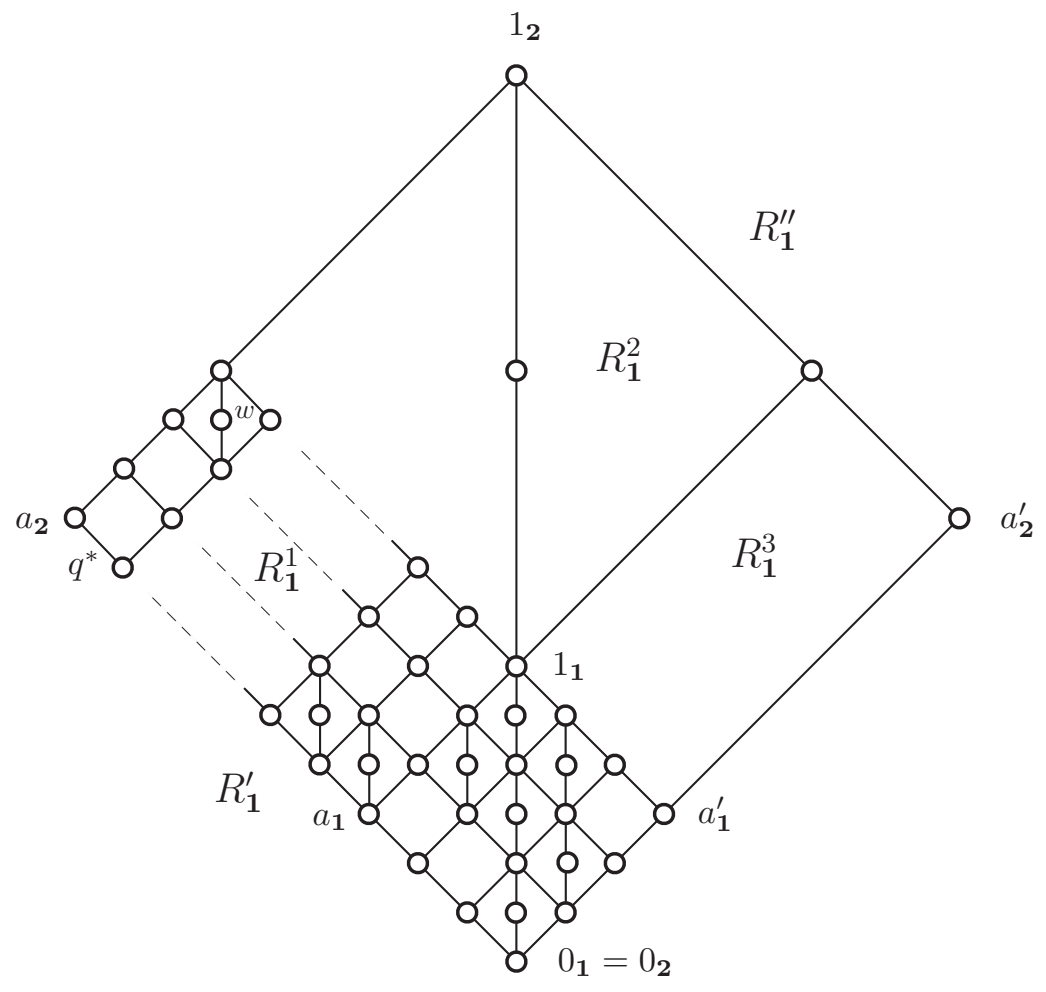

Figure 3 . The lattice $R_{2}$.

Let $p$ be a maximal element of $P$. Then for the poset $Q=P-\{p\}$, there exists a rigid modular lattice $R_{Q}$ with a dual atom $q_{Q}$ such that $\left(I_{1}\right)-\left(I_{4}\right)$ hold; in particular, there is a chain

$$
0_{Q} \leq b_{1}<c_{1} \leq b_{2}<c_{2} \leq \cdots \leq b_{n-1}<c_{n-1} \leq a_{Q}^{\prime}
$$

such that the join-irreducible congruences of $R_{Q}$ are the principal congruences $\Theta\left(b_{1}, c_{1}\right), \Theta\left(b_{2}, c_{2}\right), \ldots, \Theta\left(b_{n-1}, c_{n-1}\right)$. Let $C=\left[a_{Q}\right)$; by $\left(I_{3}\right)$ (observing that, by modularity, $\left.\left(a_{Q}^{\prime}\right] \cong\left[a_{Q}\right)\right), C$ is a well-ordered chain; it is isomorphic to $\alpha_{Q}$.

Let $q_{1}, q_{2}, \ldots, q_{r}$ be the elements of $P$ covered by $p$. Let $\Theta\left(b_{k_{1}}, c_{k_{1}}\right), \Theta\left(b_{k_{2}}, c_{k_{2}}\right)$, $\ldots, \Theta\left(b_{k_{r}}, c_{k_{r}}\right)$ be the join-irreducible congruences corresponding to $q_{1}, q_{2}, \ldots, q_{r}$, respectively. Without loss of generality, we can assume that

$$
b_{k_{1}}<c_{k_{1}} \leq b_{k_{2}}<c_{k_{2}} \leq \cdots \leq b_{k_{r}}<c_{k_{r}} .
$$

Let $D$ be the chain

$$
\left[b_{k_{1}}, c_{k_{1}}\right] \oplus\left[b_{k_{2}}, c_{k_{2}}\right] \oplus \cdots \oplus\left[b_{k_{r}}, c_{k_{r}}\right]
$$

(which is the disjoint union of the intervals in which the top element of each interval is identified with the bottom element of the next interval). For every natural number $i$, let $D_{i}$ be a chain isomorphic to $D$, and let $x^{i}$ denote the image of $x$ in $D_{i}$ under this isomorphism. Finally, we consider the ordinal sum $\mathcal{C}$ of these chains with two elements adjoined, $q^{*}$ and $1^{*}$, where $1^{*}$ is the unit element of $\mathcal{C}$ and $q^{*}$ is 
a dual atom,

$$
\mathcal{C}=D_{1} \oplus D_{2} \oplus \cdots \oplus\left\{q^{*}, 1^{*}\right\} .
$$

We apply Lemma 4 first to $U_{1}=C$ and $V_{1}=\left[b_{k_{1}}, c_{k_{1}}\right]$, to obtain the lattice $C \circledast\left[b_{k_{1}}, c_{k_{1}}\right]$ and dual ideal $\left\{c_{k_{1}}\right\} \times C(\cong C)$, and second to $U_{2}=C$ and $V_{2}=$ $\left[b_{k_{2}}, c_{k_{2}}\right]$, to obtain the lattice $C \circledast\left[b_{k_{2}}, c_{k_{2}}\right]$ and ideal $\left\{b_{k_{2}}\right\} \times C(\cong C)$, and we glue these two lattices together over the given ideal and dual ideal. We proceed similarly and glue $C \circledast\left[b_{k_{3}}, c_{k_{3}}\right]$ to the resulting lattice, and so on. In $r$ steps, we obtain a lattice $L$ with an ideal and a dual ideal both isomorphic to $C$.

Now for each $i<\omega$, we take a copy $L_{i}$ of $L$, and glue $L_{2}$ to $L_{1}, L_{3}$ to the resulting lattice, and so on, in the last step, we glue $L_{i}$. Call the lattice we obtained $\bar{L}_{i}$. Obviously, $\bar{L}_{1} \subseteq \bar{L}_{2} \subseteq \ldots \subseteq \bar{L}_{n}$, so we can take $\bigcup\left(\bar{L}_{i} \mid i<\omega\right)$. We adjoin to this lattice $\left\{q^{*}, 1^{*}\right\} \times C$ (where $\left\{q^{*}, 1^{*}\right\}$ is regarded as the two-element chain with $\left.q^{*}<1^{*}\right)$ so that

$$
\begin{aligned}
& \langle u, c\rangle \wedge\langle v, d\rangle=\langle u \wedge v, c \wedge d\rangle, \\
& \langle u, c\rangle \vee\langle v, d\rangle=\langle u \vee v, c \vee d\rangle,
\end{aligned}
$$

for $u$ and/or $v \in\left\{q^{*}, 1^{*}\right\}$ and $c, d \in C$.

We add one more element. $C$ has a dual atom $q_{C}$; the lattice $\left\{q^{*}, 1^{*}\right\} \times\left\{q_{C}, 1_{Q}\right\}$ is a cover-preserving four-element Boolean sublattice of the lattice we have constructed. We add an element $w$ so that $\left\{q^{*}, 1^{*}\right\} \times\left\{q_{C}, 1_{Q}\right\}$ with $w$ form a coverpreserving $M_{3}$. Let $R_{Q}^{1}$ denote the lattice we have just obtained.

Finally, let us glue $R_{Q}$ and $R_{Q}^{1}$ together over the dual ideal $C$ and ideal $\left\{b_{k_{1}}\right\} \times C$ $(\cong C)$ in $L_{0}$; call the resulting lattice $R_{Q}^{\prime}$.

To investigate $R_{Q}^{\prime}$, let us present a more intuitive description. Form the direct product of $\mathcal{C}$ and $C$; we shall call this the base of $R_{Q}^{1}$. Consider the interval $\left[b_{k_{j}}^{i}, c_{k_{j}}^{i}\right]$ in $\mathcal{C}$ and the corresponding interval $\left[b_{k_{j}}, c_{k_{j}}\right]$ in $C$. In the direct product $\mathcal{C} \times C$, replace

$$
\left[b_{k_{j}}^{i}, c_{k_{j}}^{i}\right] \times\left[b_{k_{j}}, c_{k_{j}}\right]
$$

with $M_{3}\left[\left[b_{k_{j}}, c_{k_{j}}\right]\right.$ so that

$$
\langle x, y\rangle \in\left[b_{k_{j}}^{i}, c_{k_{j}}^{i}\right] \times\left[b_{k_{j}}, c_{k_{j}}\right]
$$

is replaced by $\langle x, x \wedge y, y\rangle$ (see statement 5 of Lemma 2) and we add the element $w$ in the top prime square so that it form an $M_{3}$. Of course, with this definition it is not clear whether $R_{Q}^{1}$ is a lattice, whether it is modular, and what are the congruences of $R_{Q}^{1}$. It is clear, however, that this definition is the same as the more complicated one given above, so we get all these properties of the construction from Lemmas 2-4.

Take a congruence $\Psi$ of $R_{Q}^{1}$; then the restriction of $\Psi$ to the base of $R_{Q}^{1}$ is of the form $\Phi \times \Theta$, where $\Phi$ is a congruence of $\mathcal{C}$ and $\Theta$ is a congruence of $C$. It is clear from Lemmas $2-4$ that $\Phi$ and $\Theta$ uniquely determine $\Psi$. Moreover, $\Theta$ "almost" determines $\Phi$. In fact, $a \equiv b(\Phi)$ is fully determined by $\Theta$, for $a, b \in D_{1} \oplus D_{2} \oplus \ldots$. The same is true for $a, b \in\left\{q^{*}, 1^{*}\right\}$. Finally, let $a \in D_{1} \oplus D_{2} \oplus \ldots$ and $b \in\left\{q^{*}, 1^{*}\right\}$. Then $a \equiv b(\Phi)$ implies that all $D_{i}$ are collapsed by $\Phi$ from some $i$ on, and so all of $D_{1} \oplus D_{2} \oplus \cdots$ is collapsed by $\Phi$. We conclude that $R_{Q}^{1}$ has exactly one joinirreducible congruence that is not a minimal extension of a congruence from $C$, namely, $\Theta\left(\left\langle 0_{\mathcal{E}}, 0_{C}\right\rangle,\left\langle q^{*}, 0_{C}\right\rangle\right)$, and this congruence majorizes all the join-irreducible 
congruences that are minimal extensions of the congruences $\Theta\left(b_{1}, c_{1}\right), \Theta\left(b_{2}, c_{2}\right), \ldots$, $\Theta\left(b_{n-1}, c_{n-1}\right)$ of $C$ to $R_{Q}^{1}$.

By the inductive assumption $\left(I_{3}\right)$, every congruence of $R_{Q}$ is determined by its action on $C$, and so we obtain that every congruence of $R_{Q}$ can be extended to $R_{Q}^{\prime}$. Therefore, the join-irreducible congruences of $R_{Q}^{\prime}$ can be described as follows: they are the minimal extensions of the join-irreducible congruences of $R_{Q}$ to $R_{Q}^{\prime}$ and the congruence $\Theta\left(\left\langle 0_{\mathfrak{e}}, 0_{C}\right\rangle,\left\langle q^{*}, 0_{C}\right\rangle\right)$. Hence they form a poset isomorphic to $P$, and so Con $R_{Q} \cong \mathfrak{D}$, by $\left(I_{2}\right)$.

The lattice $R_{Q}^{\prime}$ does not satisfy all the inductive assumptions, so we shall define $R_{P}$ as an appropriate extension of $R_{Q}^{\prime}$.

Define the lattice $R_{Q}^{2}$ as $M_{3}[\mathrm{C}]$ and let $\mathcal{C}_{1}(\cong \mathcal{C})$ be the ideal of $M_{3}[\mathrm{C}]$ as defined in statement 3 of Lemma 2. Define the lattice $R_{Q}^{3}$ as $\left[a_{Q}^{\prime}, 1_{Q}\right] \times \mathcal{C}_{1}$ and let $\mathcal{C}_{2}$ $\left(\cong \mathcal{C}\right.$ ) be the dual ideal generated by $\left\langle 1_{Q}, 0_{\mathcal{C}_{1}}\right\rangle$ in $R_{Q}^{3}$. Let $R_{Q}^{\prime \prime}$ be the gluing of $R_{Q}^{2}$ and $R_{Q}^{3}$ over $\mathcal{C}_{1}$ and $\mathcal{C}_{2}$. Obviously, the ideal of $R_{Q}^{\prime \prime}$ generated by $\langle 1,0,0\rangle \in R_{Q}^{2}$ is isomorphic with the dual ideal $\left[a_{Q}^{\prime}\right)$ of $R_{Q}^{\prime}$. So we can glue $R_{Q}^{\prime}$ and $R_{Q}^{\prime \prime}$ together to obtain $R_{P}$.

Define $a_{P}=\left\langle 1^{*}, 0_{C}\right\rangle\left(\in R_{Q}^{1}\right)$ and let $a_{P}^{\prime}$ be its unique complement, $a_{P}^{\prime}=$ $\left\langle a_{Q}^{\prime}, 0_{\mathfrak{C}_{1}}\right\rangle\left(\in R_{Q}^{3}\right)$. The dual atom $\left\langle 1^{*}, q^{*}, q^{*}\right\rangle$ of $R_{Q}^{2}$ can serve as the dual atom of $R_{P}$. Now we verify that $R_{P}$ satisfies the conditions of Theorem 2 as well as the inductive assumptions $\left(I_{1}\right)-\left(I_{4}\right)$.

$\left(a_{P}\right]$ is a countable well-ordered chain, namely, $\alpha_{Q} \oplus \mathcal{C} \oplus \mathbf{2}$, which is a successor ordinal; the same is true of $\left(a_{P}^{\prime}\right]$.

Let $\alpha$ be an automorphism of $R_{P}$. Since $1_{Q}$ is the smallest element $x \in R_{P}$ such that there is a sublattice isomorphic to $M_{3}$ from $x$ to $1_{P}$, it follows that $1_{Q} \alpha=1_{Q}$. Since $R_{Q}$ is rigid, it follows that $x \alpha=x$, for all $x \in R_{Q} \subseteq R_{P}$.

$R_{Q}^{1}$ is built on $\mathcal{C} \times C$, and $C$ is kept fixed by $\alpha$. Therefore, $\alpha$ maps $\mathcal{C}$ into itself. But $\mathcal{C}$ is well-ordered, so $\alpha$ is the identity map on $\mathcal{C}$, and so on all of $R_{Q}^{\prime}$.

$R_{Q}^{2}=\left[1_{Q}, 1_{P}\right]$ and both these elements are fixed by $\alpha$. Next consider the three atoms in the sublattice isomorphic to $M_{3}$ stretching from $1_{Q}$ to $1_{P}:\langle 1,0,0\rangle$ is fixed because it is in $R_{Q}^{\prime} ;\langle 0,1,0\rangle$ and $\langle 0,0,1\rangle$ cannot be interchanged because $1_{Q}$ has a complement in $\left[a_{Q}^{\prime},\langle 0,0,1\rangle\right]$ but not in $\left[a_{Q}^{\prime},\langle 0,1,0\rangle\right]$. So $\alpha$ fixes $\langle 1,0,0\rangle,\langle 0,1,0\rangle$, and $\langle 0,0,1\rangle$ and also the chain $\mathcal{C}$, hence $\alpha$ fixes all of $R_{Q}^{2}$. Since we already know that in $R_{Q}^{3}$, $\alpha$ fixes $\left(1_{Q}\right]$ and $\left[1_{Q}\right)$, it follows that $\alpha$ fixes every element of $R_{Q}^{3}$. We conclude that $R_{P}$ is rigid.

It remains to verify $\left(I_{3}\right)$. In $R_{Q}$, we were given the chain

$$
0_{Q} \leq b_{1}<c_{1} \leq b_{2}<c_{2} \leq \cdots \leq b_{n-1}<c_{n-1} \leq a_{Q}^{\prime}
$$

such that the join-irreducible congruences of $R_{Q}$ are the principal congruences $\Theta\left(b_{1}, c_{1}\right), \Theta\left(b_{2}, c_{2}\right), \ldots, \Theta\left(b_{n-1}, c_{n-1}\right)$. In $R_{P}$, we extend this chain with $b_{n}=a_{Q}^{\prime}$ and $c_{n}=a_{P}^{\prime}$. Then condition $\left(I_{4}^{\prime}\right)$ is obvious.

This completes the proof of Theorem 2 .

\section{Proof of the Independence Theorem for Modular Lattices}

Now it is easy to prove the Independence Theorem for Modular Lattices. Let $\mathfrak{G}$ and $\mathfrak{D}$ be given as in the Independence Theorem. Let $S$ be constructed as in Theorem 1 for $\mathfrak{G}$ and let $R$ be the lattice in Theorem 2 constructed for $\mathfrak{D}$. We take 
the ideal $(p]$ in $S$ and the dual ideal $[q)$ in $R$; both are two-element lattices, so we can glue $S$ and $R$ together over $(p]$ and $[q)$. Let $M$ be the resulting lattice.

$M$ is a modular lattice. By statement 4 of Lemma 3 , Con $M \cong$ Con $R$, so Con $M$ is isomorphic to $\mathfrak{D}$.

Now let $\alpha$ be an automorphism of $S$, and define a map $\alpha^{\dagger}$ of $M$ into itself as follows:

$$
x \alpha^{\dagger}= \begin{cases}x \alpha, & \text { if } x \in S \\ x, & \text { if } x \in R .\end{cases}
$$

Since $p$ and $0_{S}$ are kept fixed by $\alpha$, it is evident that $\alpha^{\dagger}$ is an automorphism of $M$. Moreover, $q$ and $p$ is the only pair of elements in $M$ satisfying $q \prec p$ and $M=(p] \cup[q)$; therefore, every automorphism of $M$ acts as an automorphism on $S$ and $R$. It is now clear that every automorphism of $M$ is of the form $\alpha^{\dagger}$ for some automorphism $\alpha$ of $S$, hence, $\alpha \rightarrow \alpha^{\dagger}$ is an isomorphism between the automorphism group of $S$ and the automorphism group of $M$. It follows that the automorphism group of $M$ is isomorphic to $\mathfrak{G}$.

This completes the proof of the Independence Theorem for Modular Lattices.

\section{Arguesian lattices}

The arguesian identity (see, e.g., [5, p. 260]) is very much stronger than the modular identity; it is - for projective geometries - the lattice theoretic form of Desargues' Theorem. So it is not surprising that it is more complicated to construct an arguesian lattice than a modular lattice. In this section, we outline how to modify the lattice constructions of this paper to obtain an arguesian lattice.

Let $\mathcal{V}_{2}$ denote the quasivariety of lattices that can be embedded in a projective geometry (identified with its subspace lattice) over the two-element field. Of course, every lattice in $\mathcal{V}_{2}$ is arguesian but not conversely.

We shall prove the following:

Independence Theorem for $\mathcal{V}_{2}$. Let $\mathfrak{D}$ be a finite distributive lattice with more than one element and let $\mathfrak{G}$ be a finite group. Then there exists a lattice $A \in \mathcal{V}_{2}$ such that the congruence lattice of $A$ is isomorphic to $\mathfrak{D}$ and the automorphism group of $A$ is isomorphic to $\mathfrak{G}$.

First, we state a stronger form of Theorem 1:

Theorem 1'. Let $\mathfrak{G}$ be a finite group. Then there exists a simple lattice $S \in \mathcal{V}_{2}$ with zero $0_{S}$ and an atom $p$ such that the automorphism group of $S$ is isomorphic to $\mathfrak{G}$ and every automorphism keeps $p$ fixed.

This result was proved in [8].

Later in this section, we shall need a variant of this result, namely that there is an $S \in \mathcal{V}_{2}$ satisfying the changed requirement that the dimension of $p$ over $0_{S}$ is $\aleph_{0}$. This is easily done. Let $S$ be a sublattice of the projective geometry $V$ over the two-element field; just take another projective geometry $V^{\prime}$ over the two-element field that contains $\aleph_{0}$ copies of every point in $V$. Then $V$ is a sublattice of $V^{\prime}$ in the natural way; therefore, so is $S$, and the additional requirement for this embedding is obvious.

Second, we need the following stronger form of Theorem 2:

Theorem $2^{\prime}$. Let $\mathfrak{D}$ be a finite distributive lattice with more than one element. There exists a rigid lattice $R \in \mathcal{V}_{2}$, with unit $1_{R}$ and a dual atom $q$ such that the congruence lattice of $R$ is isomorphic to $\mathfrak{D}$ and the dimension of $1_{R}$ over $q$ is $\aleph_{0}$. 
Proof. The lattice $R$ constructed in Theorem 3 could be described as follows: $R$ is built on the direct square of a countable well-ordered chain in which some "squares" $\left[\left\langle a_{1}, b_{1}\right\rangle,\left\langle a_{2}, b_{2}\right\rangle\right]$ satisfying $\left[a_{1}, a_{2}\right] \cong\left[b_{1}, b_{2}\right]$ are "replaced" by $M_{3}\left[\left[a_{1}, a_{2}\right]\right]$.

The embedding of $R$ into $\mathcal{V}_{2}$ is based on the following lemma:

Lemma 5. Let $V$ be a vector space, let $a, b \in V$ be of dimension $\aleph_{0}$ over $a \wedge b$. Then there is a $c \in V$ such that $\{a \wedge b, a, b, c, a \vee b\}$ form a sublattice isomorphic to $M_{3}$. Let $C$ be $a$ chain in $[a \wedge b, a]$ with $a \wedge b, a \in C$. Then $\{a \wedge b, a, b, c, a \vee b\}$ and $C$ generate a sublattice in $V$ isomorphic to $M_{3}[C]$.

Proof. Let $\left\{u_{1}, u_{2}, \ldots\right\}$ be a basis of $a$ over $a \wedge b$, and $\left\{v_{1}, v_{2}, \ldots\right\}$ be a basis of $b$ over $a \wedge b$. We define $c \in V$ as the subspace generated by $a \wedge b$ and

$$
\left\{u_{1}+v_{1}, u_{2}+v_{2}, \ldots\right\} \text {. }
$$

It is now clear that $\{a \wedge b, a, b, c, a \vee b\}$ forms an $M_{3}$. The second statement follows now by a reference to statement 7 of Lemma 2 .

Now to embed $R$ into $\mathcal{V}_{2}$, take a projective geometry $V$ with a countably infinite base $B$ over the two-element field. Split $B$ into two disjoint countably infinite sets $B=B_{1} \cup B_{2}$, and map $0_{R}$ into the subspace generated by $B_{1}$. Now we split $B_{2}$ into two collections of disjoint countably infinite sets $\left\{X_{i} \mid i \in \alpha_{P}\right\}$ and $\left\{Y_{i} \mid i \in \alpha_{P}\right\}$ and represent $\alpha_{P}$ with the two chains: $x \in \alpha_{P}$ is mapped into the subspace generated by $B_{1} \cup \bigcup\left(X_{i} \mid i<x\right)$ in the first chain, and $x \in \alpha_{P}$ is mapped into the subspace generated by $B_{1} \cup \bigcup\left(Y_{i} \mid i<y\right)$ in the second chain.

Whenever we have to replace $\left[\left\langle a_{1}, b_{1}\right\rangle,\left\langle a_{2}, b_{2}\right\rangle\right]$ satisfying $\left[a_{1}, a_{2}\right] \cong\left[b_{1}, b_{2}\right]$ by $M_{3}\left[\left[a_{1}, a_{2}\right]\right]$, we apply Lemma 5 . This embeds $R$ into $V$.

To prove the Independence Theorem for $\mathcal{V}_{2}$, represent the lattice $R$ of Theorem $2^{\prime}$ with the additional requirement that the dimension of $1_{R}$ over $q$ is $\aleph_{0}$. Now represent the lattice $S$ of Theorem $1^{\prime}$ by mapping $0_{S}$ into the subspace representing $q$ and by mapping $p$ into the subspace representing $1_{R}$, and then proceed to represent $S$ as in the proof of Theorem $1^{\prime}$.

\section{REFERENCES}

[1] V. A. Baranskil, On the independence of the automorphism group and the congruence lattice for lattices, Abstracts of lectures of the 15th All-Soviet Algebraic Conference, Krasnojarsk, July 1979, vol. 1, p. 11.

[2] V. A. Baranskiŭ, Independence of lattices of congruences and groups of automorphisms of lattices, Izv. Vyssh. Uchebn. Zaved. Mat. 1984, no. 12, 12-17, 76 (Russian). English translation: Soviet Math. (Iz. VUZ) 28 (1984), no. 12, 12-19.

[3] G. Birkhoff, On groups of automorphisms (Spanish), Revista Unión Mat. Argentina 11 (1946), 155-157.

[4] G. Grätzer, General Lattice Theory, Academic Press, New York, N. Y., Birkhäuser Verlag, Basel, Akademie Verlag, Berlin, 1978.

[5] - General Lattice Theory, second edition, new appendices by the author with B. A. Davey, R. Freese, B. Ganter, M. Greferath, P. Jipsen, H. A. Priestley, H. Rose, E. T. Schmidt, S. E. Schmidt, F. Wehrung, and R. Wille. Birkhäuser Verlag, Basel, 1998. xx+663 pp. ISBN: 0-12-295750-4, ISBN: 3-7643-5239-6. Softcover edition, Birkhäuser Verlag, Basel-BostonBerlin, 2003, ISBN 3-7643-6996-5.

[6] G. Grätzer and E. T. Schmidt, On congruence lattices of lattices, Acta Math. Acad. Sci. Hungar. 13 (1962), 179-185.

[7] _ The Strong Independence Theorem for automorphism groups and congruence lattices of finite lattices. Beiträge Algebra Geom. 36 (1995), 97-108. 
[8] _ On finite automorphism groups of simple arguesian lattices, Studia Sci. Math. Hungar. 35 (1999), 247-258.

[9] A. Mitchke and R. Wille, Freie modulare Verbände $F M\left({ }_{D} M_{3}\right)$. Proceedings of the University of Houston Lattice Theory Conference (Houston, Tex., 1973), pp. 383-396. Dept. Math., Univ. Houston, Houston, Tex., 1973.

[10] E. T. Schmidt, Über die Kongruenzverbänder der Verbände, Publ. Math. Debrecen 9 (1962), 243-256.

[11] A. Urquhart, A topological representation theory for lattices, Algebra Universalis 8 (1978), $45-58$.

Department of Mathematics, University of Manitoba, Winnipeg, Man. R3T 2N2, CANADA

E-mail address: gratzer@cc.umanitoba.ca

URL: http://server.math.umanitoba.ca/homepages/gratzer/

Mathematical Institute of the Budapest University of Technology and Economics, Mǘgyetem RKP. 3, H-1521 Budapest, Hungary

E-mail address, E. T. Schmidt: schmidt@math.bme.hu

URL, E. T. Schmidt: http://www.bme.math/ ${ }^{\sim}$ schmidt/ 